\title{
Application of 3D additive manufacturing and robotics for hand prosthetic design
}

\begin{abstract}
Currently the mechanical design of hand exoprostheses are perform from computational programs by generating numerical models without theoretical considerations and mainly producing an extremely robust component (which are antiesthetic) with excessive weight, so it is necessary to produce modifications into the development of these elements taking into account Robotics, Additive Manufacturing and the use of materials with little-known applications. Additionally, by applying these kind of techniques, costs can be reduced, the manufacture process can be simpler and less time consumed, and the developed product could be more efficiently.
\end{abstract}

Keywords: robust, antiesthetic, numerical models, robotics
Volume 3 Issue 6 - 2019

\author{
Diaz León C, Urriolagoitia Sosa G, \\ Hernández Ramírez A, Romero Ángeles B, \\ Islas Jiménez DI, Martínez Reyes J, Gallegos \\ Funes FJ, Urriolagoitia Calderón GM \\ Instituto Politécnico Nacional, México
}

Correspondence: Christian Diaz León, Politécnico Nacional, Higher School of Mechanical and Electrical Engineering, Lindavista, Delegation Gustavo A. Madero, C.P. 07320, Mexico, Email christiandiazleon180@gmail.com

Received: October 15, 2019 | Published: November 19, 2019
Abbreviations: CAD, computer aided design; CAE, computer aided engineering; TPE, thermoplastic elastomer

\section{Introduction}

The beginning in the mechanical design of prosthetic elements for upper limb dates from approximately $2000 \mathrm{BC}$, which is related to a discovery found in an Egyptian mummy. Nevertheless, the prosthetic element only can served as supporting devices for the forearm. ${ }^{1}$ On the other hand, with the implementation as material of manufacture of Iron, new prostheses devices were developed, with the objective of being used in the war holding swords or shields. ${ }^{2}$ However, in the sixteenth century would be presented the first hand substitute, designed by Ambroise Pare, as a consequence would begin with the design of different prostheses with different objectives and materials for production. ${ }^{3}$ Today, the loss of the upper limb of the human body due to a congenital disease, trauma or accident, leads to several limitations into the daily activities. So, it is essential to replace the affected part with a prosthesis, either aesthetic or functional type. ${ }^{4}$ However, now days, there are prosthesis designed and manufacture that exceed 70 000 euros in their production, as well as hand substitutes made from low-cost additive manufacturing. ${ }^{5}$ Therefore, the replacement of this part of the body is more accessible due to the combination of various areas such as Engineering, Biomechanics and Robotics, that allow to mechanical design functional prostheses by the application of mathematical foundations. By the application of these sciences, it is possible to predict the proper functionality of these elements. Also, the practice and improvement of new technologies, such as free-source additive manufacturing and the use of unconventional materials, such as thermostable polymers, provides an optimal product. As a result, prostheses can be designed to be capable of mimicking some of the most characteristic movements of this limb, ${ }^{6}$ such as opposition of the thumb with one of the other fingers, as well as particular features that possess the physiognomy of the hand, causing that this type of $t$ prosthesis gives a more aesthetic look. Finally, the research presented in this work concludes in a design of a robotic arms which comprehends a functional hand prosthesis with five fingers with their corresponding phalanges. Additional and as an advantage of the product developed, this prosthetic device was produce by $3 \mathrm{D}$ printing and their movement capacities were developed and corroborated by Direct Kinematics sciences.

\section{Proposal ${ }^{7}$}

The use of robotic fundaments as the methodology described by Denevit \& Hertenberg ${ }^{8}$ allows to perform analysis of the movement functionality of the hand and/or elements that act as fingers as if it were a robotic manipulator. This evaluation emerges from a homogeneous transformation matrix, which has the aim of determine the spatial and angular positions that the prosthetic element can provide to the patient. This mathematical results can be corroborated by the application of specialize computational programs capable of plotting mathematical results (Figure 1). This kind of analysis are performed in a two steps procedure, the first involving the link representing the thumb due to the multiple movements it can perform and the second analysis will correspond to the remaining fingers, in this research only taking two fingers; where both finger are considered as index (performing the same movement).

Subsequently, the use of Computer Aided Design $(C A D)$ or Computer Aided Engineering (CAE) computational programs make it possible to sketch the prosthesis with each one of all of its elements (Figure 2). The prosthesis device was produce by considering average dimensions of the Mexican male population, as well as, physiophonic features contained in the human hand. With the model developed in SolidWorks it is exported as an STL file, in order to be applied in a free source 3D printer and to produce the prosthesis by Additive Manufacture process, using a Thermoplastic Elastomer (TPE) as an input material, due to its thermo-mechanical properties. The manufacture of the prosthetic device is obtained by using an interface user-machine software, which will provide a visualization of the printing process, as well as the estimated printing time and the number of layers to be occupied (Figure 3) After the 23hours, that take the element to be printed, a flexible hand substitute capable of 
performing opposition movements with three of the four long fingers was obtained. In addition, also it is included the transradial base on which the servo motor is placed, from where the cable joints are connected to transmit the movement to the prosthesis elements. In this same location a logical programmer which by means of electrodes communicated to the patient's arm to transmit myolecteric signals to reproduce the desired movements by the user (Figure 4).

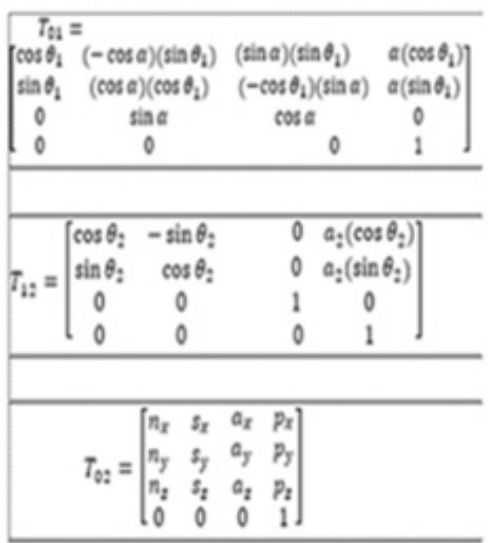

Figure I Equations and movement for hand thumb.

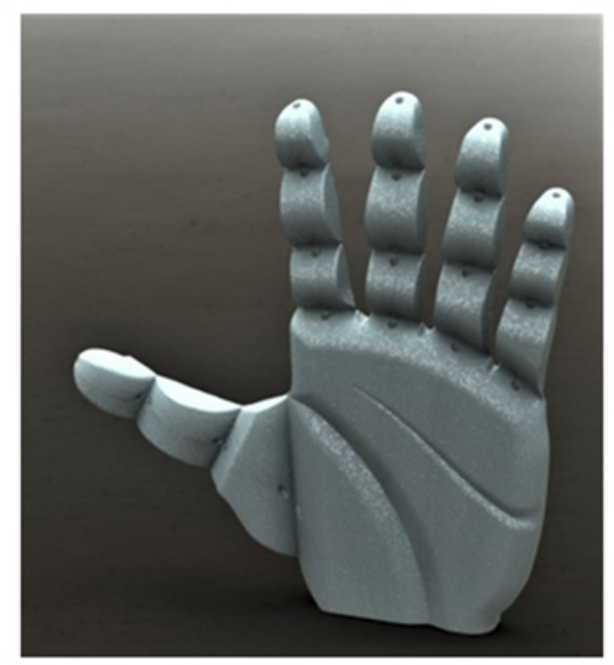

Figure 2 Prosthesis scheme of the human hand produce by Solid Works.

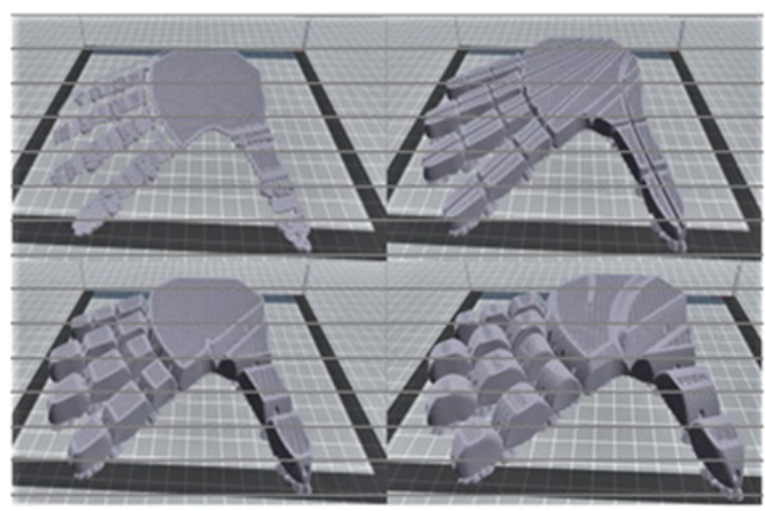

Figure 3 Printing process.
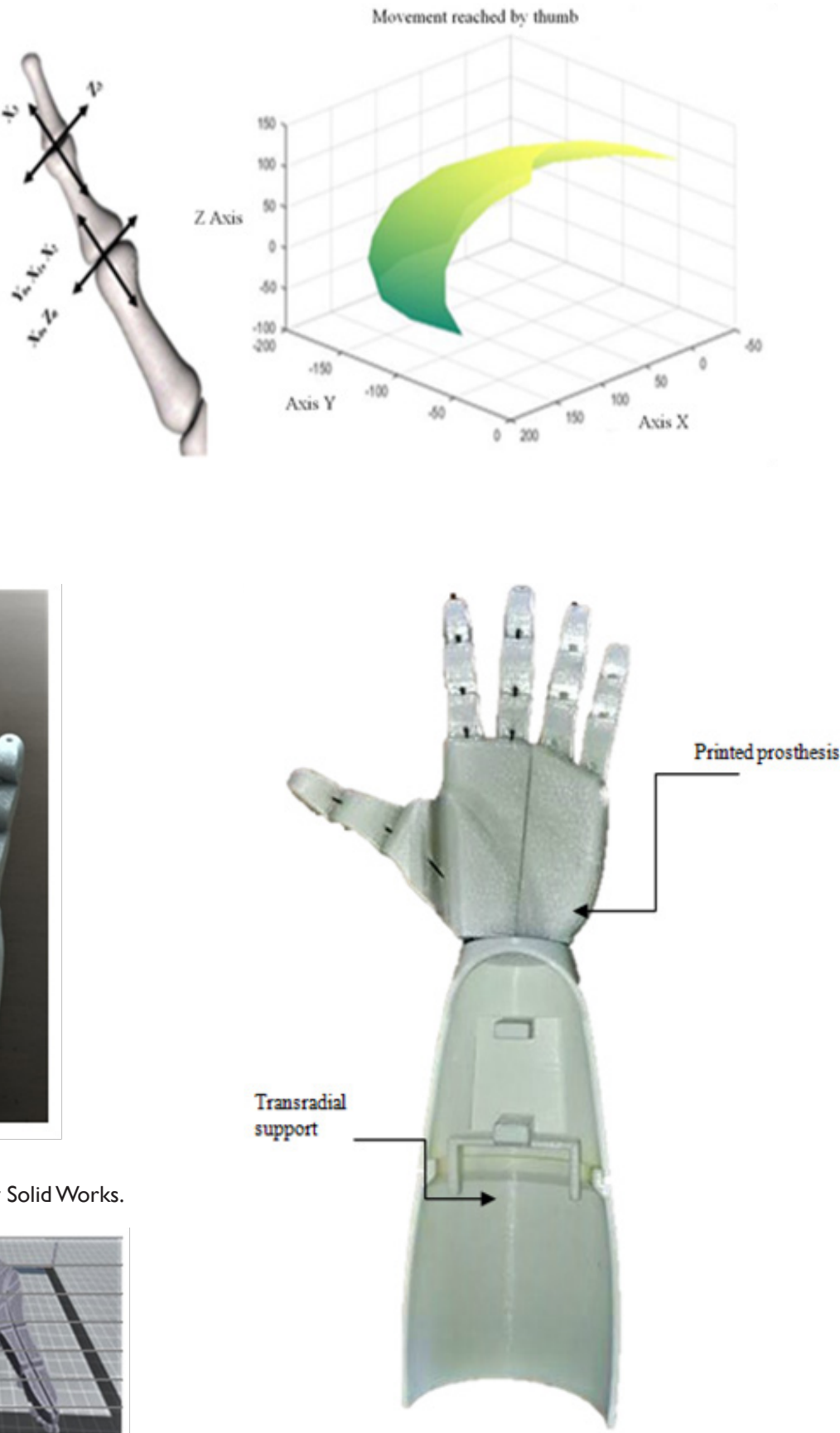

Figure 4 Final prosthesis.

\section{Discussion}

The development of hand exoprostheses must be constantly changing due to advances in science, as well as, in the technologies currently used, in addition to the exploration of new materials that achieve a more aesthetic aspect or seek the possibility to involve them in the processes currently used to obtain such elements. 


\section{Conclusion}

The unifying various areas in the design of exoprostheses results in elements capable of mimicking some functions of the actual limbs by understanding their behavior before and after obtaining the final product. In addition to the use of unconventional materials in the application of prostheses such as Medical Grade Steel or Titanium, it can open up the possibility of exploring the potential that Thermostable Elastomers can provide in the design of prostheses together with new technologies developed. Today the use of Additive Manufacturing is known worldwide, however, the application of this technology has not been discovered in its entirety because it is considered as new, therefore it is an area of research with many growth opportunities that can be explored along with other established areas of science.

\section{Acknowledgements}

The authors thank to the Instituto Politécnico Nacional and the Consejo Nacional de Ciencia y Tecnología the support provided in the realization of this work.

\section{Conflicts of interest}

The author declares that there is no conflict of interest.

\section{Funding}

None.

\section{References}

1. Dorado González JM. Robotics and smart prostheses. Digital University Magazine, 2004;6(1):4-6.

2. Murphy WB. Spare Parts: From Peg Legs to Gene 20 Splices, Brookfield, 2001. p. 8-12.

3. Loaiza JL, Arzola N. Evolution and trends in the development of hand prosthesis. Dyna. 2011;78(169):1-7.

4. Smurr LM, Gulick K, Yancosek K, et al. Managing the upper extremity amputee: A protocol for success. J Hand Ther. 2008;21(8):160-176.

5. Huang $H$. The development on a new biomechatronic prosthetic hand based on under-actuated mechanism. IEEE/RSJ International Conference on Intelligent Robots and Systems. 2006. p. 3791-3796.

6. Romero Ángeles B, Hernández Campos D, Urriolagoitia Sosa G, et al. Design and manufacture of a forearm prosthesis by plastic 3D impression for a patient whit trasradial amputation applied for strum a guitar. Engineering Design Applications, Ed. Springer, 2019. p. 97-121.

7. Diaz León C. Diseño de una Prótesi de Mano con Implementación de Falanges, M. Sc. Thesis, Instituto Politécnico Nacional, SEPI ESIME Zacatenco, 2018. p. 44-62.

8. Denevit J, Hartenberg RS. A kinematic notation for lower-pair mechanisms based on matrices. Journal of Applied Mechanics. $1955 ; 77(2): 215-221$. 